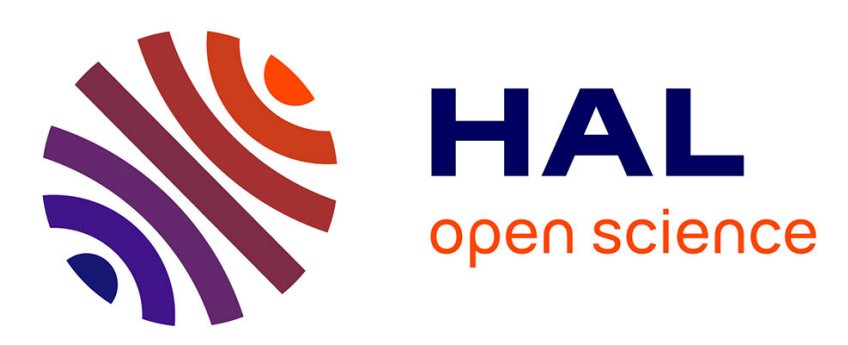

\title{
Analytic Calculation of Eddy Currents in the Slots of Electrical Machines: Application to Cage Rotor Induction Motors
}

\author{
Thierry Lubin, Smail Mezani, Abderrezak Rezzoug
}

\section{To cite this version:}

Thierry Lubin, Smail Mezani, Abderrezak Rezzoug. Analytic Calculation of Eddy Currents in the Slots of Electrical Machines: Application to Cage Rotor Induction Motors. IEEE Transactions on Magnetics, 2011, 47 (11), pp.4650-4659. 10.1109/TMAG.2011.2157167 . hal-00640594

\author{
HAL Id: hal-00640594 \\ https://hal.science/hal-00640594
}

Submitted on 13 Nov 2011

HAL is a multi-disciplinary open access archive for the deposit and dissemination of scientific research documents, whether they are published or not. The documents may come from teaching and research institutions in France or abroad, or from public or private research centers.
L'archive ouverte pluridisciplinaire HAL, est destinée au dépôt et à la diffusion de documents scientifiques de niveau recherche, publiés ou non, émanant des établissements d'enseignement et de recherche français ou étrangers, des laboratoires publics ou privés. 


\title{
Analytic Calculation of Eddy Currents in the Slots of Electrical Machines: Application to Cage Rotor Induction Motors
}

\author{
Thierry Lubin, Smail Mezani, and Abderrezak Rezzoug \\ Groupe de Recherche en Electrotechnique et Electronique de Nancy, \\ University Henri Poincaré, Nancy, FRANCE
}

This paper describes a two-dimensional analytic model for predicting the induced currents within conducting material placed in the slots of electrical machines. Slot-embedded conductors are subjected to a rotating magnetic field including space harmonics. The analytical method is based on the resolution of time-harmonic homogeneous Helmholtz's equation in polar coordinates by the separation of variables method. The global solution is obtained using boundary and continuity conditions between the different subdomains (rotor slots and air-gap). The proposed model is applied for magnetic field and torque calculations in a rotor cage induction motor under steady state condition. The analytical results are verified with those obtained from finite element analyses.

Index Terms-Eddy currents, analytical solution, diffusion equation, rotor bars, induction motor.

\section{INTRODUCTION}

$\mathrm{A}$ CCURATE modeling of eddy current effects is important to design electrical machines and especially to calculate the torque-speed curve of induction motors. Eddy-currents can be evaluated by numerical or analytical methods. With numerical methods [1]-[5], complex geometric shapes (as rotor bars in induction motor) and non-linear effects (saturation of the magnetic parts) can be taken into account. However, these methods are computer time consuming and are not suitable for the first step of design stage of electrical machines. To be fast and efficient, analytical methods suppose simple geometries (conducting plates or cylinders) and linear magnetic behavior [6-7]. Due to their low computational time, they are often preferred for the first evaluations of electrical motors performances and for design optimization.

Two-dimensional (2D) analytical models for induced currents prediction in induction motors (IM) have been proposed in the literature. They deal with solid-rotor topology (cageless rotor) which present cylindrical symmetry [8]-[12]. The induced currents distribution in the rotor (conducting cylinder) is obtained by solving the magnetic diffusion equation in polar coordinates. Analytical models are also proposed for the evaluation of slot impedance of a rectangular conductor placed in a semi-closed slot [13-14]. However, the models are developed for a single rotor bar (with imposed net current), so the interaction between neighbouring bars is ignored. These models are well suited for the determination of equivalent circuit parameters of IM.

Exact analytical models for the magnetic field prediction in the slots of electrical machines have been proposed recently [15]-[18]. However, they deal with permanent magnet machines and are based on the magnetostatic approach which neglects the eddy-current reaction field. To the author's knowledge, there has not been anything reported in the literature on 2D exact analytic models for the prediction of current density induced in the rotor bars of squirrel cage

Manuscript received January 4, 2011. Corresponding author: Thierry Lubin. (e-mail: Thierry.lubin@green.uhp-nancy.fr).

Digital Object Identifier inserted by IEEE induction motor.

In this paper, we present an original analytical model for eddy-currents computation within conducting material placed in rotor slots of electrical machines and subjected to a rotating magnetic field. Laplace's and Helmholtz's equations are solved respectively in the air-gap and in the rotor slots regions. The global solution is obtained using boundary and interface conditions between the different regions. The proposed analytical method is used to investigate the eddy current distribution in the rotor bars of an induction motor.

\section{PROBLEM DESCRIPTION AND ASSUMPTIONS}

\section{A. Problem Description}

The cross-section of the studied machine is shown in Fig. 1. The rotor contains $Q$ slots $(Q=6$ in Fig. 1$)$ with conducting material (rotor bars) of electrical conductivity $\sigma$ and magnetic permeability $\mu_{0}$. We consider that the rotor bars are permanently shorted to each other through end rings. The geometrical parameters are the inner radius of the rotor slots $R_{l}$, the radius of the rotor surface $R_{2}$ and the stator bore radius $R_{3}$. The slot opening angle is $\beta$.

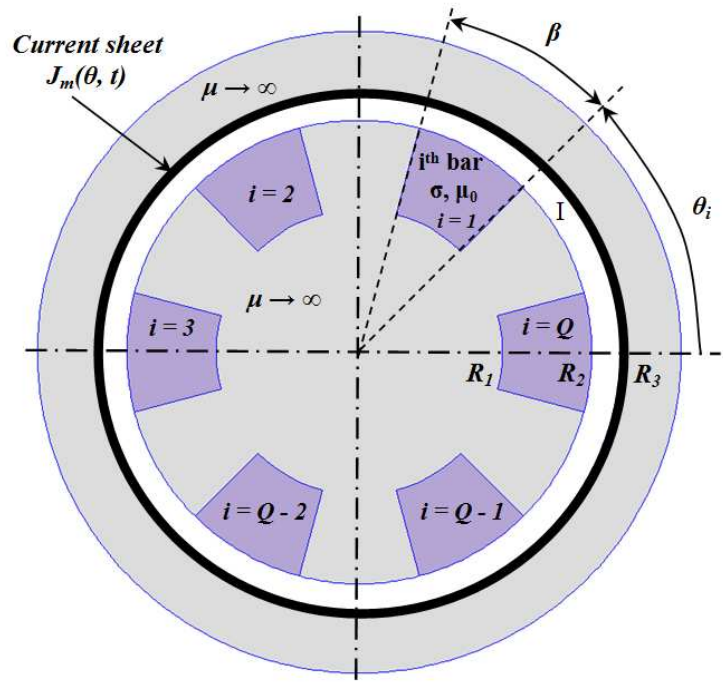

Fig. 1. Geometry of the studied model (with $Q=6$ rotor bars). 
In a frame linked to the rotor, the angular position of the $i$-th rotor bar is defined as

$$
\theta_{i}=-\frac{\beta}{2}+\frac{2 i \pi}{Q} \quad \text { with } \quad 1 \leq i \leq Q
$$

In order to simplify the theoretical analysis, we assume that the source term is represented by a current sheet distributed over the stator inner radius $\left(\mathrm{r}=R_{3}\right)$. It is well-known that the stator ampere-conductor can be approximated by an equivalent current sheet disposed over the slot openings. If the stator currents are sinusoidal in time, the expression of the current sheet $(\mathrm{A} /$ meter $)$ for the $m$-th space harmonic component is

$$
J_{m}\left(\theta_{s}, t\right)=J_{m} \cos \left(m p \theta_{s}-\omega_{s} t\right)
$$

where $J_{m}$ is the peak value of the $m$-th harmonic component, $p$ is the number of pole pairs, $\theta_{s}$ is the angular displacement on the stator bore, $\omega_{s}$ is the angular frequency of the stator supply, $t$ is time.

For a balanced three-phase stator windings distribution with an integer number of slot per poles and per phase, the $m$-th space harmonics component is given by

$$
m=1 \pm 6 l \quad \text { where } \quad l=0,1,2,3 \ldots .
$$

The peak value of the $m$-th harmonic component of the current sheet is

$$
J_{m}=\frac{3 N_{p h} I \sqrt{2}}{\pi R_{3}} K_{w m}
$$

where $N_{p h}$ is the number of series turns per phase and $I$ is the stator RMS current. The winding factor $K_{w m}$ associated with the $m$-th harmonic is given by

$$
K_{w m}=\sin (|m| \pi / 2) \sin (|m| \lambda / 2) \frac{\sin (|m| \pi / 6)}{q \sin (|m| \pi / 6 q)} \frac{\sin (|m| \varepsilon / 2)}{|m| \varepsilon / 2}
$$

where $q$ is the number of slots per pole per phase, $\lambda$ is the coil pitch angle, $\varepsilon$ is the width of the slot opening in radians ( $\lambda$ and $\varepsilon$ are electrical angles).

Each harmonic component of the current sheet produces a rotating magnetic field which rotates forward $(\mathrm{m}=1,7,13 \ldots)$ or backward $(m=-5,-11, \ldots)$ with respect to the stator with an angular mechanical speed given by

$$
\Omega_{s m}=\frac{\omega_{s}}{m p}
$$

\section{B. Assumptions}

The model presented here is based on the following main assumptions

- The machine is infinitely long (end effects are neglected).

- Stator and rotor iron cores are infinitely permeable (saturation effect is neglected) and the stator core is slotless.

- The rotor iron circuit is perfectly laminated so that eddy currents are negligible.
- The rotor slots have radial sides as shown in Fig.1.

- The current density has only one component along $z$-axis.

- The stator windings are fed with sinusoidal current of angular frequency $\omega_{s}$.

- The angular velocity of the rotor noted $\Omega$ is constant.

- The electrical conductivity of the rotor bars is constant.

As shown in Fig.1, the whole domain is divided into $1+Q$ subdomains: the airgap subdomain (regions I) which has an annular shape and $Q$ rotor bars subdomains (regions $i$ ). The $i$ th rotor bar subdomain is shown in Fig. 2. To describe the problem, a magnetic vector potential formulation is used in $2 \mathrm{D}$ polar coordinates. According to the adopted assumptions, the magnetic vector potential has only one component along the $z$ direction and only depends on the $r$ and $\theta$ coordinates. The magnetic vector potential in the different subdomains is noted as

$$
\begin{array}{lr}
\boldsymbol{A}_{\boldsymbol{I}}=A_{I}(r, \theta, t) \boldsymbol{e}_{z} & \text { for the air-gap domain } \\
\boldsymbol{A}_{\boldsymbol{i}}=A_{i}(r, \theta, t) \boldsymbol{e}_{z} & \text { for the } i \text {-th rotor bar domain }
\end{array}
$$

\section{Current Sheet Expression in the Rotor Reference Frame}

If the rotor moves with a constant angular velocity $\Omega$, the current sheet expression (2) can be transformed from the stator reference frame (angle $\theta_{s}$ ) to the rotor reference frame (angle $\theta$ ) such that

$$
\theta_{s}=\theta+\Omega t
$$

which leads to

$$
J_{m}(\theta, t)=J_{m} \cos \left(m p \theta-\omega_{r m} t\right)
$$

where $\omega_{r m}$ is the angular frequency of the rotor currents induced by the $m$-th harmonic rotating field. Its expression is given by

$$
\omega_{r m}=(1-m(1-s)) \omega_{s} \quad \text { with } \quad s=1-\frac{\Omega}{\Omega_{s 1}}
$$

$s$ is the rotor slip for the fundamental component of the rotating field ( $m=1$ ) and $\Omega_{s 1}$ is given by (6).

In the next subsections, the magnetic vector potentials in the air-gap and rotor slots sub-domains will be computed in the rotor reference frame for each space harmonic component. According to the form of the source term (8), the magnetic vector potentials in the whole space vary sinusoidally with time at an angular frequency $\omega_{r m}$. Therefore, it is convenient to use the complex notation

$$
\begin{aligned}
& A_{I}(r, \theta, t)=\Re\left\{\overline{A_{I}}(r, \theta) e^{j \omega_{r m} t}\right\} \\
& A_{i}(r, \theta, t)=\Re\left\{\overline{A_{i}}(r, \theta) e^{j \omega_{r m} t}\right\}
\end{aligned}
$$

where $\mathfrak{R}$ denotes the real part of the complex number and $j=\sqrt{-1}$. Using complex notation, the $m$-th harmonic component of the current sheet (8) becomes

$$
J_{m}(\theta, t)=\Re\left\{\overline{J_{m}}(\theta) e^{j \omega_{r m} t}\right\} \text { with } \overline{J_{m}}(\theta)=J_{m} e^{-j m p \theta}
$$




\section{ANALYTICAL SOLUTIONS}

\section{A. Solution of Helmholtz's Equation in the i-th Rotor Bar}

The $i$-th rotor bar sub-domain and the associated boundary conditions are shown in Fig. 2. From the quasi-static Maxwell's equations, we have to solve the complex Helmholtz equation (13) in a domain of inner radius $R_{1}$ and outer radius $R_{2}$ delimited by the angles $\theta_{i}$ and $\theta_{i}+\beta$

$$
\frac{\partial^{2} \overline{A_{i}}}{\partial r^{2}}+\frac{1}{r} \frac{\partial \overline{A_{i}}}{\partial r}+\frac{1}{r^{2}} \frac{\partial^{2} \overline{A_{i}}}{\partial \theta^{2}}=j \omega_{r m} \sigma \mu_{0} \overline{A_{i}}
$$

The tangential component of the magnetic field at the sides and at the bottom of the rotor bars is zero (the iron is infinitely permeable). In terms of magnetic vector potential, the boundary conditions are

$$
\begin{aligned}
& \left.\frac{\partial \overline{A_{i}}}{\partial \theta}\right|_{\theta=\theta_{i}}=0 \quad \text { and }\left.\quad \frac{\partial \overline{A_{i}}}{\partial \theta}\right|_{\theta=\theta_{i}+\beta}=0 \\
& \left.\frac{\partial \overline{A_{i}}}{\partial r}\right|_{r=R_{1}}=0
\end{aligned}
$$

The continuity condition between the $i$-th rotor bar and the air-gap leads to

$$
\overline{A_{i}}\left(R_{2}, \theta\right)=\overline{A_{I}}\left(R_{2}, \theta\right)
$$

The general solution of (13) can be found by using the method of the separation of variables. The magnetic vector potential is expressed as the product of two functions, each of them having only one variable

$$
\overline{A_{i}}(r, \theta)=\overline{\rho_{i}}(r) \overline{\Theta_{i}}(\theta)
$$

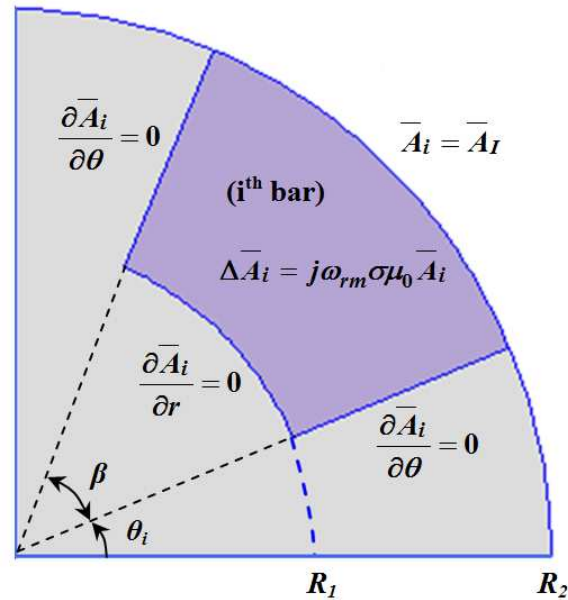

Fig. 2. i-th rotor bar domain with its boundary conditions.
Substituting (17) into (13), we obtain two ordinary differential equations where $\lambda$ is the separation constant [19]

$$
\begin{gathered}
\overline{\Theta_{i}}{ }^{\prime \prime}+\lambda \overline{\Theta_{i}}=0 \\
r^{2} \overline{\rho_{i}}{ }^{\prime}+r \overline{\rho_{i}}{ }^{\prime}+\left(\alpha^{2} r^{2}-\lambda\right) \overline{\rho_{i}}=0
\end{gathered}
$$

where $\alpha^{2}=-j \omega_{r m} \sigma \mu_{0},(19)$ is a Bessel differential equation.

Using (17), the boundary conditions (14) become

$$
\bar{\Theta}_{i}^{\prime}\left(\theta_{i}\right)=0 \quad \text { and } \quad \bar{\Theta}_{i}{ }^{\prime}\left(\theta_{i}+\beta\right)=0
$$

We are confronted to the problem of finding the values of the parameter $\lambda$ for which non-trivial solutions of (18) satisfying the boundary conditions (20) exist. This is a standard Sturm-Liouville problem [19]. The eigenvalues of the problem (18), (20) are

$$
\begin{aligned}
& \lambda_{0}=0 \\
& \lambda_{k}=\left(\frac{k \pi}{\beta}\right)^{2} \text { with } k=1,2,3, \ldots . .
\end{aligned}
$$

The eigenfunctions corresponding to $\lambda_{0}$ and $\lambda_{k}$ are given by

$$
\begin{aligned}
& \overline{\Theta_{i 0}}(\theta)=1 \\
& \overline{\Theta_{i k}}(\theta)=\cos \left(\frac{k \pi}{\beta}\left(\theta-\theta_{i}\right)\right)
\end{aligned}
$$

For $\lambda_{0}$ and $\lambda_{k}$, the general solutions of (19) are

$$
\begin{aligned}
& \overline{\rho_{i 0}}(r)=\overline{A_{0}^{i}} J_{0}(\alpha r)+\overline{B_{0}^{i}} Y_{0}(\alpha r) \\
& \overline{\rho_{i k}}(r)=\overline{A_{k}^{i}} J_{k \pi / \beta}(\alpha r)+\overline{B_{k}^{i}} Y_{k \pi / \beta}(\alpha r)
\end{aligned}
$$

where $\overline{A_{0}^{i}}, \overline{B_{0}^{i}}, \overline{A_{k}^{i}}$ and $\overline{B_{k}^{i}}$ are complex integration constants, $J_{k \pi / \beta}$ is the Bessel function of the first kind and order $k \pi / \beta$ and $Y_{k \pi / \beta}$ is the Bessel function of the second kind.

Writing the general solution as a linear combination of the previous solutions, one gets

$$
\begin{aligned}
& \overline{A_{i}}(r, \theta)=\overline{A_{0}^{i}} J_{0}(\alpha r)+\overline{B_{0}^{i}} Y_{0}(\alpha r) \\
& +\sum_{k=1}^{\infty}\left(\overline{A_{k}^{i}} J_{k \pi / \beta}(\alpha r)+\overline{B_{k}^{i}} Y_{k \pi / \beta}(\alpha r)\right) \cos \left(\frac{k \pi}{\beta}\left(\theta-\theta_{i}\right)\right)
\end{aligned}
$$

Considering the boundary condition (15) and the continuity condition (16), the general solution of the magnetic vector potential in the $i$-th rotor bar domain can be rewritten as 


$$
\begin{aligned}
& \overline{A_{i}}(r, \theta)=\overline{C_{0}^{i}}\left(\frac{J_{0}(\alpha r)-\overline{F_{1}} Y_{0}(\alpha r)}{J_{0}\left(\alpha R_{2}\right)-\overline{F_{1}} Y_{0}\left(\alpha R_{2}\right)}\right) \\
& +\sum_{k=1}^{\infty} \overline{C_{k}^{i}} \frac{J_{k \pi / \beta}(\alpha r)-\overline{F_{k}} Y_{k \pi / \beta}(\alpha r)}{J_{k \pi / \beta}\left(\alpha R_{2}\right)-\overline{F_{k}} Y_{k \pi / \beta}\left(\alpha R_{2}\right)} \cos \left(\frac{k \pi}{\beta}\left(\theta-\theta_{i}\right)\right)
\end{aligned}
$$

where $\overline{C_{0}^{i}}$ and $\overline{C_{k}^{i}}$ are new integration constants and

$$
\begin{gathered}
\overline{F_{1}}=\frac{J_{1}\left(\alpha R_{1}\right)}{Y_{1}\left(\alpha R_{1}\right)} \\
\overline{F_{k}}=\frac{-\alpha J_{1+k \pi / \beta}\left(\alpha R_{1}\right)+\frac{k \pi}{\beta R_{1}} J_{k \pi / \beta}\left(\alpha R_{1}\right)}{-\alpha Y_{1+k \pi / \beta}\left(\alpha R_{1}\right)+\frac{k \pi}{\beta R_{1}} Y_{k \pi / \beta}\left(\alpha R_{1}\right)}
\end{gathered}
$$

The integration constants $\overline{C_{0}^{i}}$ and $\overline{C_{k}^{i}}$ are determined using a Fourier series expansion of the air-gap magnetic vector potential $\overline{A_{I}}(r, \theta)$ over the slot interval $\left[\theta_{i}, \theta_{i}+\beta\right]$ at $r=R_{2}$.

$$
\begin{aligned}
& \overline{C_{0}^{i}}=\frac{1}{\beta} \int_{\theta_{i}}^{\theta_{i}+\beta} \overline{A_{I}}\left(R_{2}, \theta\right) d \theta \\
& \overline{C_{k}^{i}}=\frac{2}{\beta} \int_{\theta_{i}}^{\theta_{i}+\beta} \overline{A_{I}}\left(R_{2}, \theta\right) \cos \left(\frac{k \pi}{\beta}\left(\theta-\theta_{i}\right)\right) d \theta
\end{aligned}
$$

The expressions for the integration constants $\overline{C_{0}^{i}}$ and $\overline{C_{k}^{i}}$ are developed in the appendix.

\section{B. Solution of Laplace's Equation in the Air-Gap}

The air-gap domain and the associated boundary conditions are shown in Fig. 3. We have to solve the Laplace equation in an annulus of inner radius $R_{2}$ and outer radius $R_{3}$

$$
\frac{\partial^{2} \overline{A_{I}}}{\partial r^{2}}+\frac{1}{r} \frac{\partial \overline{A_{I}}}{\partial r}+\frac{1}{r^{2}} \frac{\partial^{2} \overline{A_{I}}}{\partial \theta^{2}}=0
$$

Considering the current sheet $\overline{J_{m}}(\theta)$ (12) and an infinite permeability of the stator back iron, the boundary condition at $r=R_{3}$ is

$$
\left.\frac{\partial \overline{A_{I}}}{\partial r}\right|_{r=R_{3}}=\mu_{0} \overline{J_{m}}(\theta)
$$

where $\mu_{0}$ is the vacuum permeability.

Due to the presence of the rotor slots, the boundary condition at $r=R_{2}$ is more complex. It can be written as follows [18]

$$
\left.\frac{\partial \overline{A_{I}}}{\partial r}\right|_{r=R_{2}}=\bar{f}(\theta)
$$

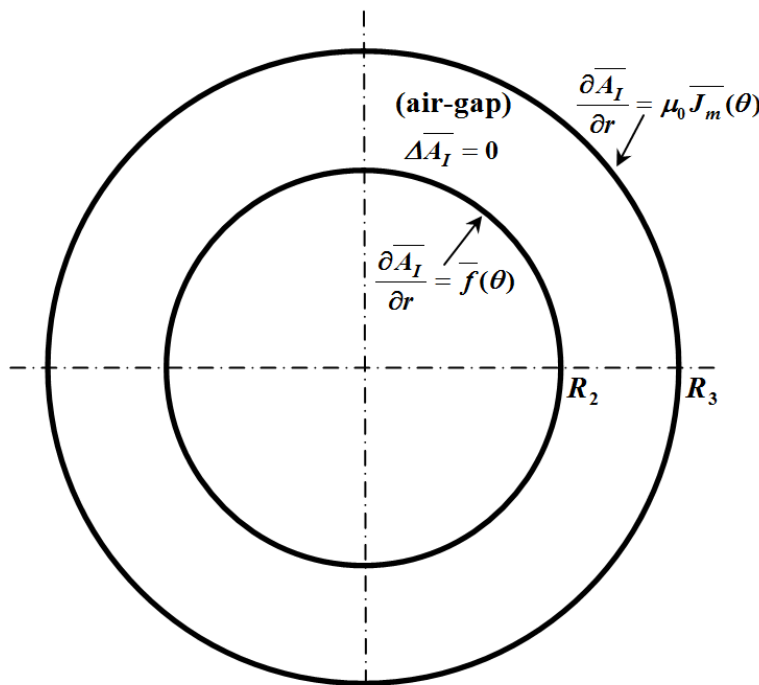

Fig. 3. Air-gap domain with its boundary conditions.

with

$$
\bar{f}(\theta)= \begin{cases}\left.\frac{\partial \overline{A_{i}}}{\partial r}\right|_{r=R_{2}} & \forall \theta \in\left[\theta_{i}, \theta_{i}+\beta\right] \\ 0 & \text { elsewhere }\end{cases}
$$

where $\overline{A_{i}}(r, \theta)$ is the magnetic vector potential in the $i$ th rotor bar given by (28).

Taking into account the boundary conditions (34) and (35), the general solution of the magnetic vector potential in the airgap can be written as

$$
\begin{aligned}
& \overline{A_{I}}(r, \theta)=\overline{A_{0}^{l}} \\
& +\sum_{n=1}^{\infty}\left(\overline{A_{n}^{I}} \frac{R_{2}}{n} \frac{P_{n}\left(r, R_{3}\right)}{E_{n}\left(R_{2}, R_{3}\right)}+\overline{B_{n}^{l}} \frac{R_{3}}{n} \frac{P_{n}\left(r, R_{2}\right)}{E_{n}\left(R_{3}, R_{2}\right)}\right) \cos (n \theta) \\
& +\sum_{n=1}^{\infty}\left(\overline{C_{n}^{l}} \frac{R_{2}}{n} \frac{P_{n}\left(r, R_{3}\right)}{E_{n}\left(R_{2}, R_{3}\right)}+\overline{D_{n}^{l}} \frac{R_{3}}{n} \frac{P_{n}\left(r, R_{2}\right)}{E_{n}\left(R_{3}, R_{2}\right)}\right) \sin (n \theta)
\end{aligned}
$$

where $n$ is a positive integer, the functions $P_{n}\left(r, R_{3}\right)$ and $E_{n}\left(R_{2}, R_{3}\right)$ are given by

$$
\begin{aligned}
& P_{n}\left(r, R_{3}\right)=\left(\frac{r}{R_{3}}\right)^{n}+\left(\frac{R_{3}}{r}\right)^{n} \\
& E_{n}\left(R_{2}, R_{3}\right)=\left(\frac{R_{2}}{R_{3}}\right)^{n}-\left(\frac{R_{3}}{R_{2}}\right)^{n}
\end{aligned}
$$

The complex integration constants $\overline{A_{n}^{I}}, \overline{B_{n}^{I}}, \overline{C_{n}^{I}}$ and $\overline{D_{n}^{I}}$ are determined using Fourier series expansions of $\mu_{0} \overline{J_{m}}(\theta)$ and $\bar{f}(\theta)$ over the air-gap interval $[0,2 \pi]$ 


$$
\begin{aligned}
& \overline{A_{n}^{I}}=\frac{2}{2 \pi} \int_{0}^{2 \pi} \bar{f}(\theta) \cos (n \theta) d \theta \\
& \overline{B_{n}^{I}}=\frac{2}{2 \pi} \int_{0}^{2 \pi} \mu_{0} \overline{J_{m}}(\theta) \cos (n \theta) d \theta \\
& \overline{C_{n}^{I}}=\frac{2}{2 \pi} \int_{0}^{2 \pi} \bar{f}(\theta) \sin (n \theta) d \theta \\
& \overline{D_{n}^{I}}=\frac{2}{2 \pi} \int_{0}^{2 \pi} \mu_{0} \overline{J_{m}}(\theta) \sin (n \theta) d \theta
\end{aligned}
$$

Taking into account (36), the coefficients (40) and (42) can be rewritten as follow

$$
\begin{aligned}
& \overline{A_{n}^{I}}=\left.\frac{2}{2 \pi} \sum_{i=1}^{Q} \int_{0}^{2 \pi} \frac{\partial \overline{A_{i}}}{\partial r}\right|_{r=R_{2}} \cos (n \theta) d \theta \\
& \overline{C_{n}^{I}}=\left.\frac{2}{2 \pi} \sum_{i=1}^{Q} \int_{0}^{2 \pi} \frac{\partial \overline{A_{i}}}{\partial r}\right|_{r=R_{2}} \sin (n \theta) d \theta
\end{aligned}
$$

The expressions for the integration constants $\overline{A_{n}^{I}}, \overline{B_{n}^{I}}, \overline{C_{n}^{I}}$ and $\overline{D_{n}^{I}}$ are developed in the appendix.

The Laplace equation (33) with the Neumann boundary conditions (34) and (35) must verify [19]

$$
\left.\int_{0}^{2 \pi} \frac{\partial \overline{A_{I}}}{\partial r}\right|_{R_{2}} R_{2} d \theta-\left.\int_{0}^{2 \pi} \frac{\partial \overline{A_{I}}}{\partial r}\right|_{R_{3}} R_{3} d \theta=0
$$

The treatment of (46) yields to the following relations between the coefficient $\overline{C_{0}^{i}}$ defined in (31)

$$
\sum_{i=1}^{Q} \overline{C_{0}^{i}}=0
$$

The radial and tangential flux density distribution in the airgap can be deduced from the magnetic vector potential (37) by

$$
\overline{B_{I r}}=\frac{1}{r} \frac{\partial \overline{A_{I}}}{\partial \theta} \quad \overline{B_{I \theta}}=-\frac{\partial \overline{A_{I}}}{\partial r}
$$

\section{EDDY-CURRENTS AND TORQUE EXPRESSIONS}

\section{A. Current in the $i$-th Rotor Bar}

The current density induced in the $i$-th rotor bar by the $m$-th space harmonic of the rotating magnetic field is given by

$$
\overline{J_{i}}(r, \theta)=-j \omega_{r m} \sigma \overline{A_{i}}(r, \theta)
$$

The current flowing through the $i$-th rotor bar is determined by

$$
\overline{I_{i}}=\int_{R_{1}}^{R_{2}} \int_{\theta_{i}}^{\theta_{i}+\beta} \overline{J_{i}}(r, \theta) r d r d \theta
$$

Considering (28) and (49), (50) yields to

$$
\overline{I_{i}}=\frac{-j \overline{C_{0}^{i}} \sigma \omega_{r m} \beta}{J_{0}\left(\alpha R_{2}\right)-\overline{F_{1}} Y_{0}\left(\alpha R_{2}\right)} \int_{R_{1}}^{R_{2}} r\left(J_{0}(\alpha r)-\overline{F_{1}} Y_{0}(\alpha r)\right) d r
$$

The development of (51) leads to

$$
\overline{I_{i}}=\frac{j \overline{C_{0}^{i}} \sigma \omega_{r m} \beta R_{2}}{\alpha}\left(\frac{J_{1}\left(\alpha R_{1}\right) Y_{1}\left(\alpha R_{2}\right)-J_{1}\left(\alpha R_{2}\right) Y_{1}\left(\alpha R_{1}\right)}{J_{0}\left(\alpha R_{2}\right) Y_{1}\left(\alpha R_{1}\right)-J_{1}\left(\alpha R_{1}\right) Y_{0}\left(\alpha R_{2}\right)}\right)
$$

where $\alpha^{2}=-j \omega_{r m} \sigma \mu_{0}$. With this relation, it is worth noting that the condition (47) imposes that the sum of the currents in the rotor bars is zero.

\section{B. Electromagnetic Torque}

The electromagnetic torque is obtained using the Maxwell stress tensor. A circle of radius $\mathrm{R}_{\mathrm{e}}$ in the air-gap is taken as the integration path. The electromagnetic torque due to the $m$-th space harmonics of the rotating field is expressed as follows

$$
T_{e}=\frac{L R_{e}^{2}}{\mu_{0}} \int_{0}^{2 \pi} \frac{1}{2} \Re\left[\overline{B_{I r}}\left(R_{e}, \theta\right){\overline{B_{I \theta}}}^{*}\left(R_{e}, \theta\right)\right] d \theta
$$

where $L$ is the axial length of the motor. Substituting (48) into (53), the analytical expression for the electromagnetic torque becomes

$$
T_{e}=\frac{\pi L R_{e}^{2}}{\mu_{0}} \sum_{n=1}^{\infty}\left(\bar{W}_{n} \cdot \bar{X}_{n}^{*}+\bar{Y}_{n} \cdot \bar{Z}_{n}^{*}\right)
$$

with

$$
\begin{aligned}
& \overline{W_{n}}=-\overline{A_{n}^{I}} \frac{R_{2}}{R_{e}} \frac{P_{n}\left(R_{e}, R_{3}\right)}{E_{n}\left(R_{2}, R_{3}\right)}-\overline{B_{n}^{I}} \frac{R_{3}}{R_{e}} \frac{P_{n}\left(R_{e}, R_{2}\right)}{E_{n}\left(R_{3}, R_{2}\right)} \\
& \overline{X_{n}}=-\overline{C_{n}^{I}} \frac{R_{2}}{R_{e}} \frac{E_{n}\left(R_{e}, R_{3}\right)}{E_{n}\left(R_{2}, R_{3}\right)}-\overline{D_{n}^{I}} \frac{R_{3}}{R_{e}} \frac{E_{n}\left(R_{e}, R_{2}\right)}{E_{n}\left(R_{3}, R_{2}\right)} \\
& \overline{Y_{n}}=\overline{C_{n}^{I}} \frac{R_{2}}{R_{e}} \frac{P_{n}\left(R_{e}, R_{3}\right)}{E_{n}\left(R_{2}, R_{3}\right)}+\overline{D_{n}^{I}} \frac{R_{3}}{R_{e}} \frac{P_{n}\left(R_{e}, R_{2}\right)}{E_{n}\left(R_{3}, R_{2}\right)} \\
& \overline{Z_{n}}=-\overline{A_{n}^{I}} \frac{R_{2}}{R_{e}} \frac{E_{n}\left(R_{e}, R_{3}\right)}{E_{n}\left(R_{2}, R_{3}\right)}-\overline{B_{n}^{I}} \frac{R_{3}}{R_{e}} \frac{E_{n}\left(R_{e}, R_{2}\right)}{E_{n}\left(R_{3}, R_{2}\right)}
\end{aligned}
$$

and ${\overline{X_{n}}}^{*}$ is the complex conjugate of $\overline{X_{n}}$.

where $\overline{A_{i}}(r, \theta)$ is given by (28). 


\section{RESULTS AND DISCUSIONS}

In order to validate the proposed model, the analytical results have been compared with those obtained from $2 \mathrm{D}$ timeharmonic FEM simulations [20]. The finite-element solutions were obtained by imposing the natural Neumann condition along the iron boundaries, as in the analytical study. The mesh in the different subdomains has been refined until convergent results are obtained. The analytical and finite-element simulations are carried out for the whole motor cross section. Obviously, if periodicity of the magnetic field distribution exists, it can be taken into account.

The geometrical parameters given in Table 1 are used in the simulation studies. These parameters have been chosen arbitrary. The objective of this subsection is not to study a real induction motor but to show the effectiveness of the proposed analytical method. The analytical solutions in the airgap and in the rotor bars regions have been computed with a finite number of harmonic terms $N$ and $K$ as indicated in Table I.

For the equivalent current sheet, we consider a full pitch stator winding $(\lambda=\pi)$ with a number of slots per pole per phase $q=1$. Moreover, we suppose that the stator slot opening $\varepsilon$ is small. So, the value of the winding factor (5) is equal to 1 for each harmonic component (the peak values $J_{m}$ of the current sheet harmonics are all the same as it is indicated in Table I). As the peak value of the stator current sheet is imposed whatever the slip, the results presented here correspond to an induction motor fed from a constant current source.

\section{A. Results for the fundamental component of the stator current sheet}

We first consider the fundamental component of the current sheet

$$
J_{1}(\theta, t)=J_{1} \cos \left(p \theta-\omega_{r 1} t\right) \quad \text { with } \quad \omega_{r 1}=s \omega_{s}
$$

Figure 4 shows the flux lines in the machine under no-load condition $(\mathrm{s} \approx 0)$. The corresponding radial and tangential components of the flux density distribution in the middle of the air-gap are shown in Fig. 5. The effects of the rotor slotting can be clearly observed in these figures. A good accordance can be seen between the finite element and the analytical computations.

TABLE I

PARAMETERS OF THE MODEL

\begin{tabular}{lll}
\hline \hline \multirow{2}{*}{ Symbol } & \multicolumn{1}{c}{ Quantity } & \multicolumn{1}{c}{ value } \\
\hline$R_{1}$ & Inner radius of the rotor slots & $4 \mathrm{~cm}$ \\
$R_{2}$ & Radius of the rotor surface & $6 \mathrm{~cm}$ \\
$R_{3}$ & Stator bore radius & $6.3 \mathrm{~cm}$ \\
$L$ & Axial length & $20 \mathrm{~cm}$ \\
$Q$ & Number of rotor bars & 18 \\
$\beta$ & Rotor slot opening & $0.6 \pi / Q \mathrm{rad}$ \\
$\sigma$ & Electrical conductivity of the rotor bars (Al.) & $35.10^{6} \mathrm{~S} / \mathrm{m}$ \\
$p$ & Pole-pairs number of the current sheet & 2 \\
$J_{m}$ & Peak value of the $m$-th harmonic component of & $8.10^{4} \mathrm{~A} / \mathrm{m}$ \\
& the current sheet & $314 \mathrm{rad} / \mathrm{s}$ \\
$\omega_{s}$ & Angular frequency of the stator supply & 50 \\
$\mathrm{~N}$ & Number of harmonics used for magnetic field & \\
& calculation in the air-gap region & 5 \\
$K$ & Number of harmonics used for magnetic field & 5 \\
\hline \hline
\end{tabular}

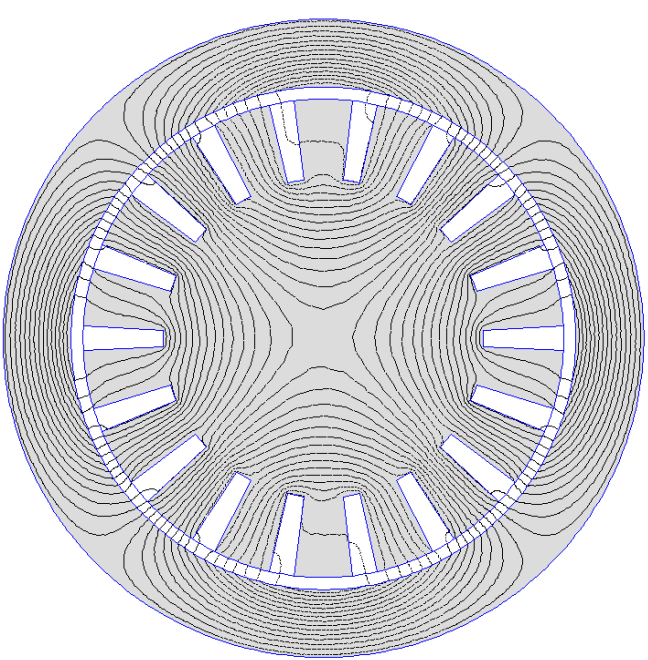

Fig. 4. Flux lines for no-load condition $(s \approx 0)$ and $m=1$ (fundamental component of the current sheet) obtained with FEM.

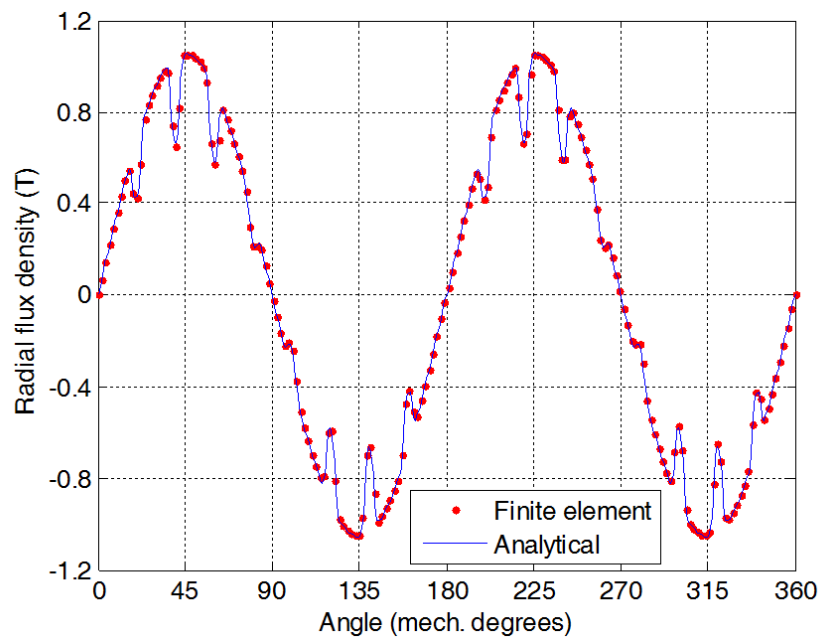

(a)

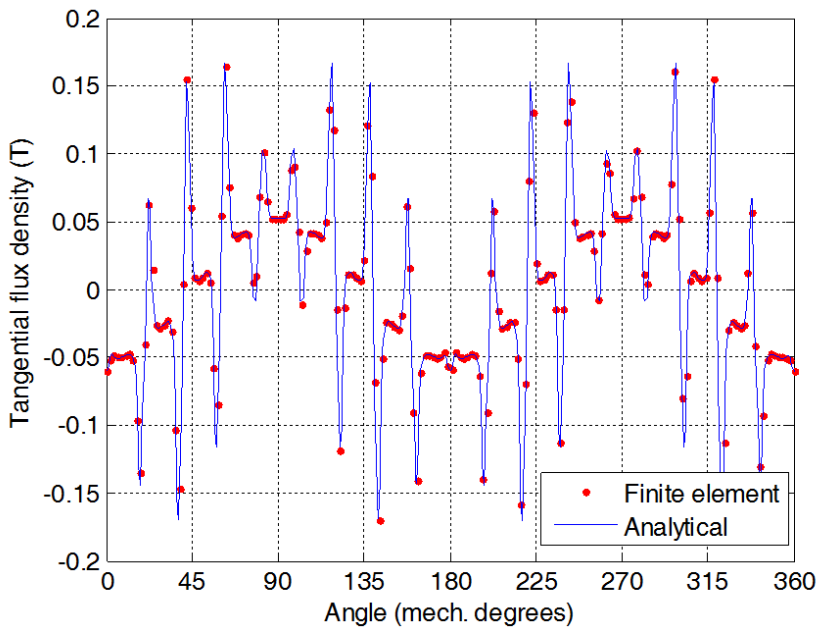

(b)

Fig. 5. Radial (a) and tangential (b) component of the flux density in the middle of the airgap for no load condition $(s \approx 0)$ and $m=1$ (fundamental component of the current sheet). 
The flux lines and the eddy-current distribution within the rotor bars for locked rotor condition $(s=1)$ are shown in Fig.6. Due to the induced current reaction, one can observe that the magnetic field is rejected at the surface of the rotor. Fig. 7 presents the corresponding radial and tangential flux density distribution in the middle of the airgap for $s=1$. Compared to the no-load results of Fig.5, one can see clearly the influence of the eddy-current reaction field on both the radial and the tangential flux densities (as expected, the radial component of the flux density decreases for locked rotor condition).

The current density distribution from the bottom to the top of a rotor bar (slot $Q=18 ; \theta=0$ ) is given in Fig. 8. Due to the well-known skin effect, the current density tends to flow at the outer surface of the rotor bar. The real part of the total current in each rotor bar computed with (52) is shown in Fig. 9.

Comparison between numerical and analytical prediction of the torque-slip curve for the fundamental component of the current sheet is given in Fig. 10 for slip values ranging from -0.2 to +0.2 .

It can be seen from Fig. 7 to Fig. 10 that the results obtained with the proposed analytical method are consistent with those obtained by FEM.

\section{B. Results for the $5^{\text {th }}$ harmonics of the stator current sheet}

To show the effectiveness of the proposed model for the modeling of space harmonics effects, we consider now the $5^{\text {th }}$ harmonic of the current sheet

$$
J_{5}(\theta, t)=J_{5} \cos \left(5 p \theta+\omega_{r 5} t\right) \text { with } \omega_{r 5}=(6-5 s) \omega_{s}
$$

Figure 11 shows the flux lines (with 10 pole-pairs) and the eddy-current distribution in the rotor bars for locked rotor condition $(s=1)$. The corresponding radial and tangential components of the flux density distribution are shown in Fig. 12. The current density distribution along a rotor bar $(Q=18)$ and the total current in each rotor are given respectively in Fig. 13 and Fig. 14.

As it is known, the fifth harmonic field rotates in a direction opposite to the one of the rotor with an angular speed

$$
\Omega_{s 5}=-\frac{\Omega_{s}}{5}
$$

Therefore, the fifth harmonic has its synchronism for a slip value

$$
s=\frac{6}{5}=1.2
$$

The torque due to the $5^{\text {th }}$ harmonic is shown in Fig.15. As expected, the torque is null for $\mathrm{s}=1.2$. The operating region for which $\mathrm{s}<1.2$ corresponds to a generator mode (negative torque).

The results presented in Fig. 12 to Fig. 15 show that the analytical model is able to predict the effect of space harmonics with a very good accuracy.

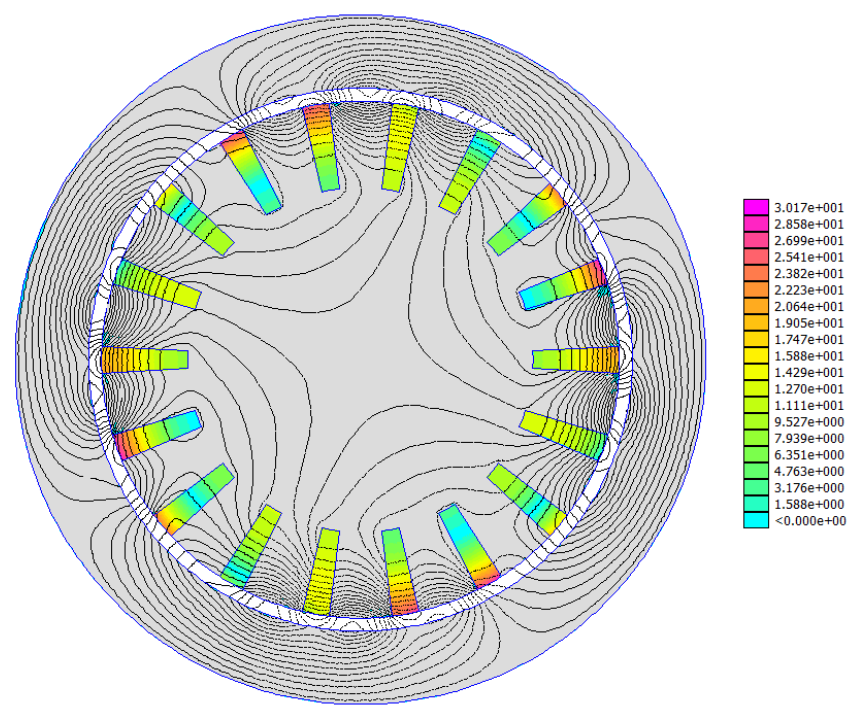

Fig. 6. Flux lines and eddy-current distribution $\left(\mathrm{A} / \mathrm{mm}^{2}\right)$ for $s=1$ (locked rotor condition) and $m=1$ (fundamental component of the current sheet) obtained with FEM.

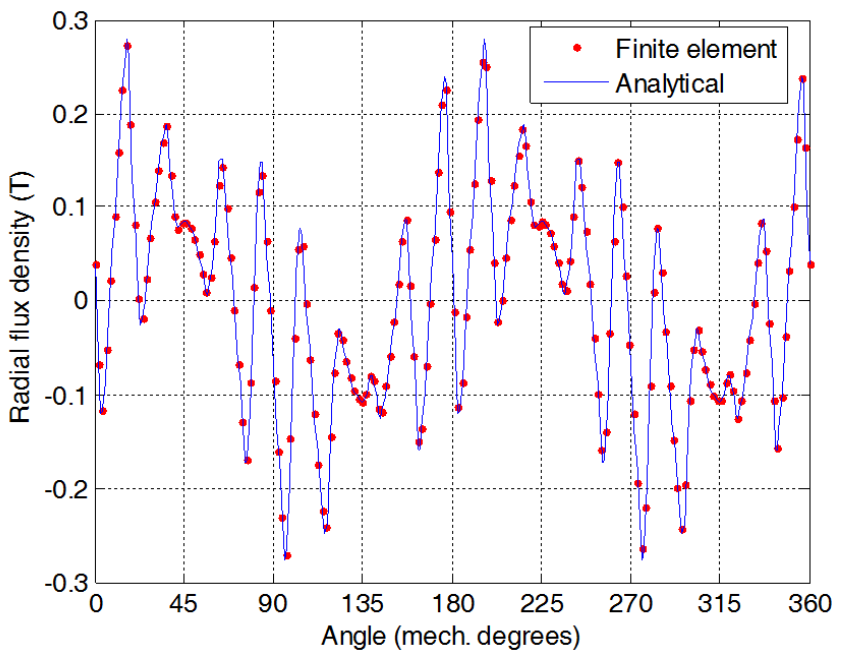

(a)

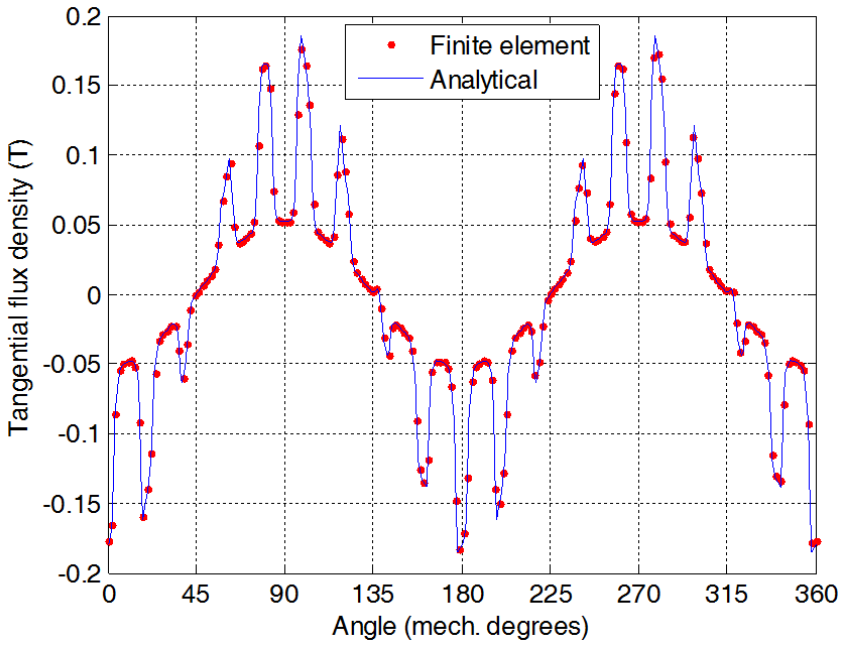

(b)

Fig. 7. Radial (a) and tangential (b) component of the flux density in the middle of the airgap for locked rotor condition $(s=1)$ and $m=1$ (fundamental component of the current sheet). 


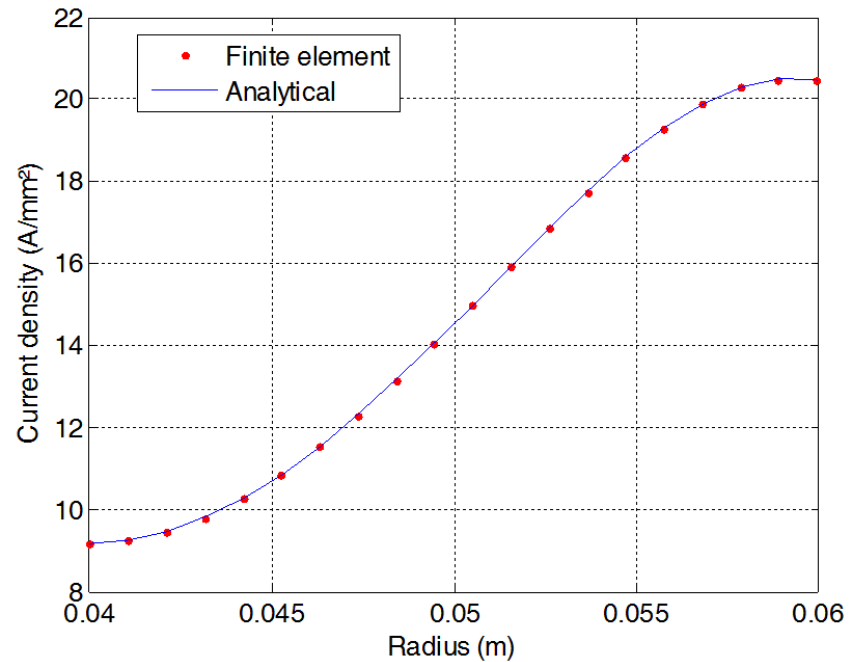

Fig. 8. Current density distribution along the $18^{\text {th }}$ rotor bar $(\theta=0)$ for locked rotor condition $(s=1)$ and $m=1$.

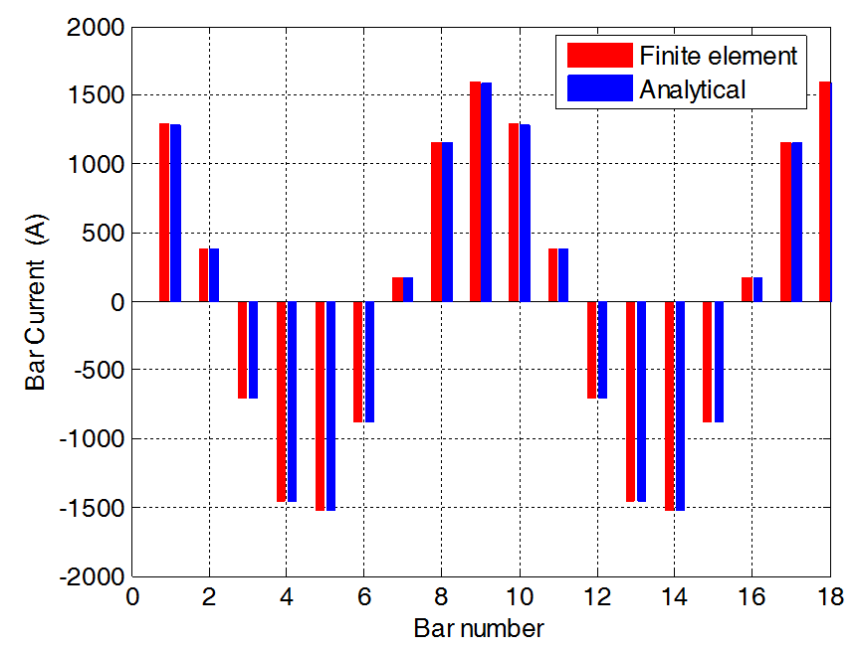

Fig. 9. Current distribution in the rotor bars (real part) for locked roto condition $(s=1)$ and $m=1$.

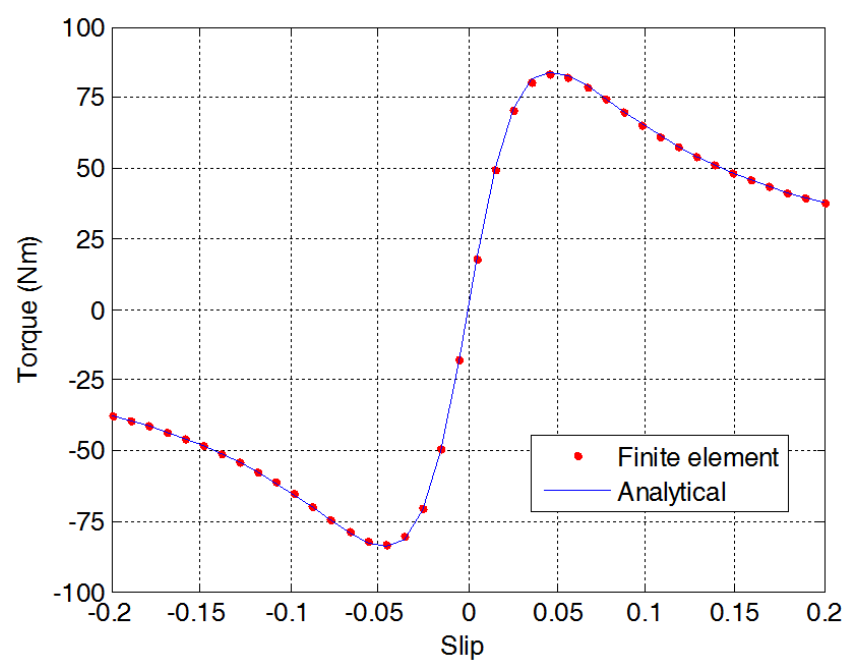

Fig. 10. Torque-slip curve for the fundamental component of the stator current sheet $(m=1)$.

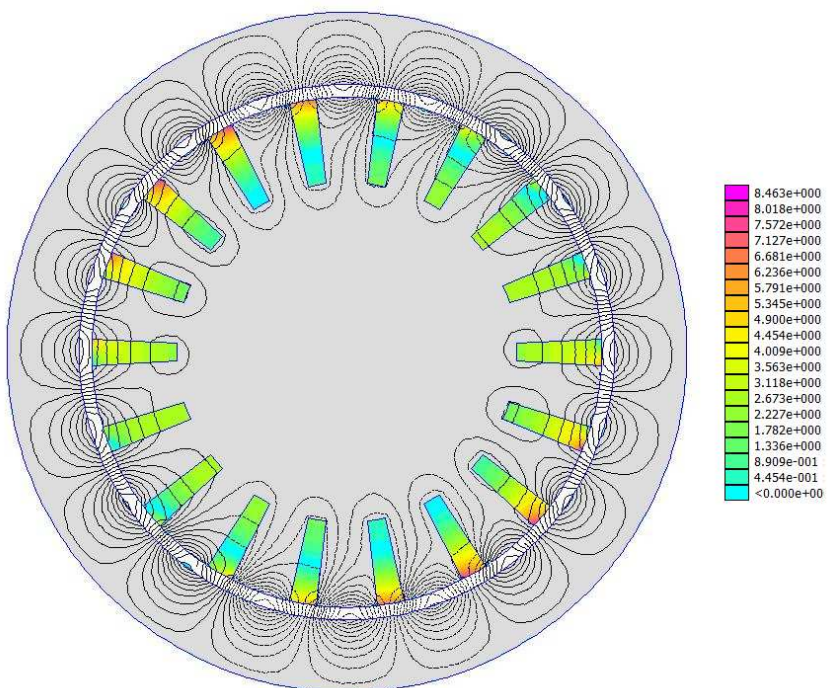

Fig. 11. Flux lines and eddy-current distribution $\left(\mathrm{A} / \mathrm{mm}^{2}\right)$ for $s=1$ (locked rotor condition) and $m=-5$ ( $5^{\text {th }}$ harmonic component of the current sheet) obtained with FEM.

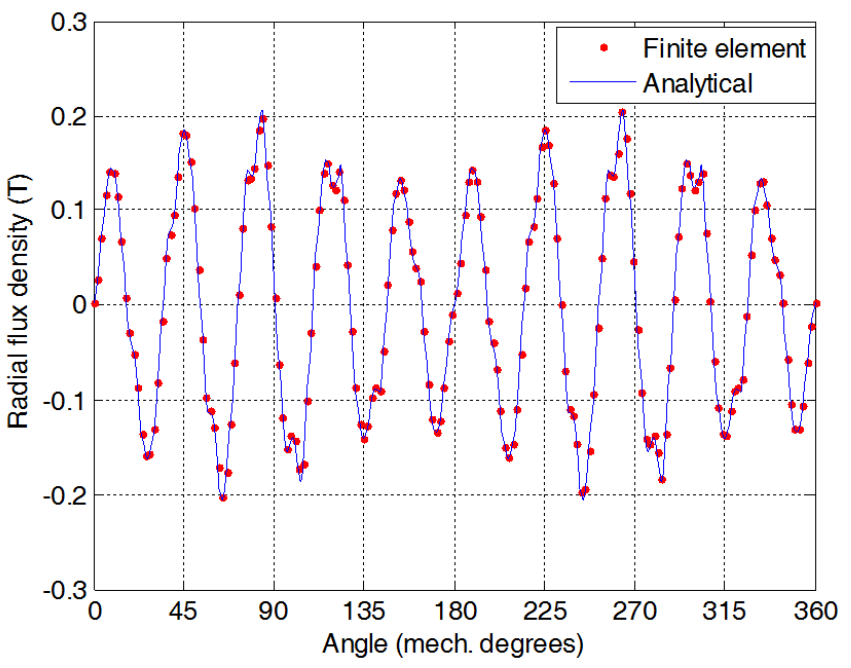

(a)

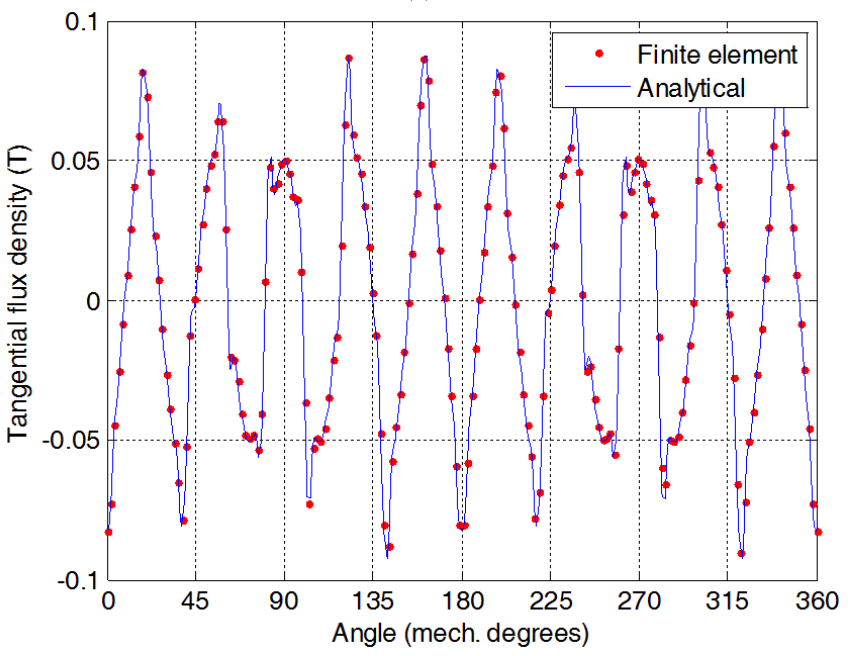

(b)

Fig. 12. Radial (a) and tangential (b) component of the flux density in the middle of the airgap for locked rotor condition $(s=1)$ and $m=-5$. 


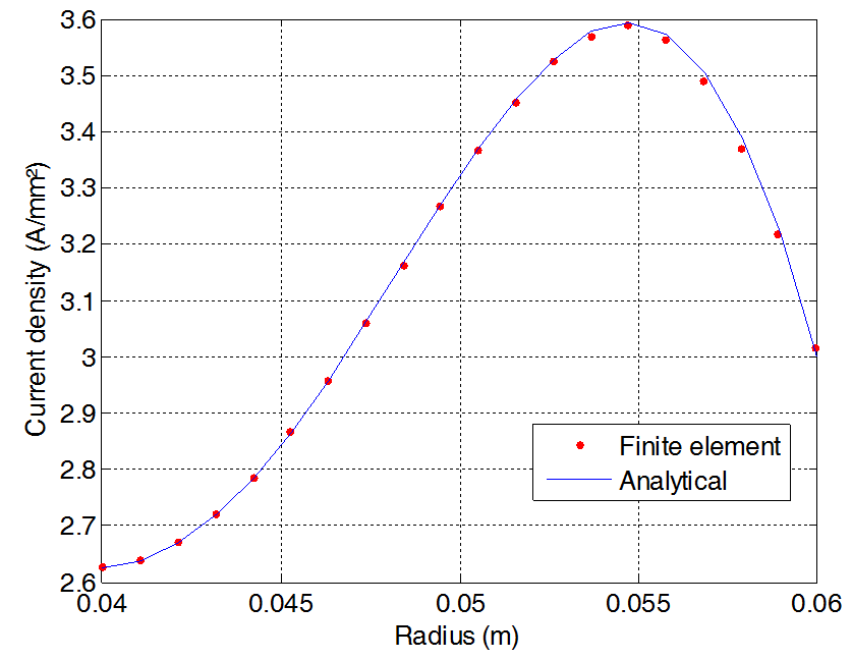

Fig. 13. Current density distribution along the $18^{\text {th }}$ rotor bar $(\theta=0)$ for locked rotor condition $(s=1)$ and $m=-5$.

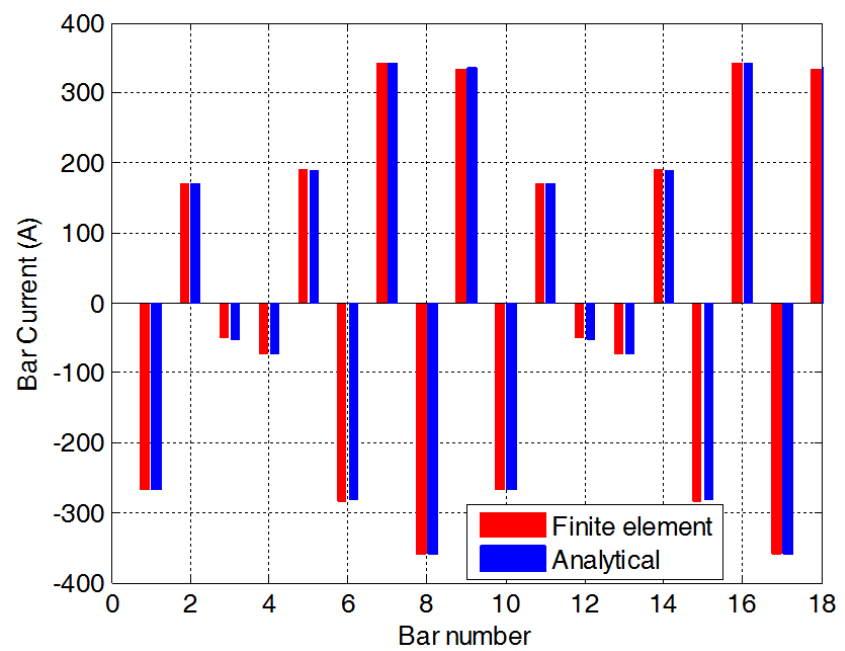

Fig. 14. Current distribution in the rotor bars (real part) for locked rotor condition $(s=1)$ and $m=-5$.

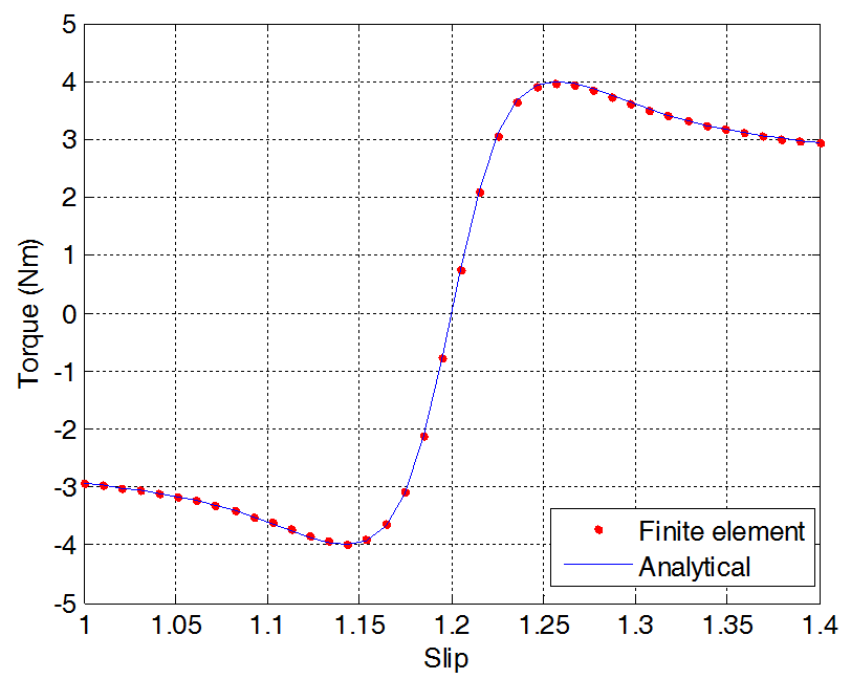

Fig. 15. Torque-slip characteristic for the $5^{\text {th }}$ harmonic component of the stator current sheet $(m=-5)$.

\section{Torque with $5^{\text {th }}$ and $7^{\text {th }}$ space Harmonic Effects}

We now use the analytical model to compute the torque for the fundamental, the $5^{\text {th }}$ and the $7^{\text {th }}$ space harmonic components of the current sheet. For a given slip value, the number of computations needed is equal to the number of considered space harmonics. Indeed, the angular frequency is different for each harmonic (9).

The torque produced by the fundamental component and the $5^{\text {th }}$ and $7^{\text {th }}$ harmonic are shown in Fig. 16. The total torque is the sum of all the components. We can observe the wellknown parasitic effects of the $5^{\text {th }}$ and $7^{\text {th }}$ space harmonics on the torque-slip characteristic of an induction machine.

In order to have a good precision in the analytical torque prediction (harmonic 1 of Fig.16), it is sufficient, for the studied machine, to take the fundamental component in the airgap region $(N=1)$ and the two first harmonic terms in the rotor bars region $(K=2)$. For a given slip value, the computation time is about $70 \mathrm{~ms}$ with the analytical model whereas the linear FEM takes about $2 \mathrm{~s}$ for a mesh of 16200 elements. The analytical computations being much faster, the presented model can advantageously be used in a preliminary design of induction motors.

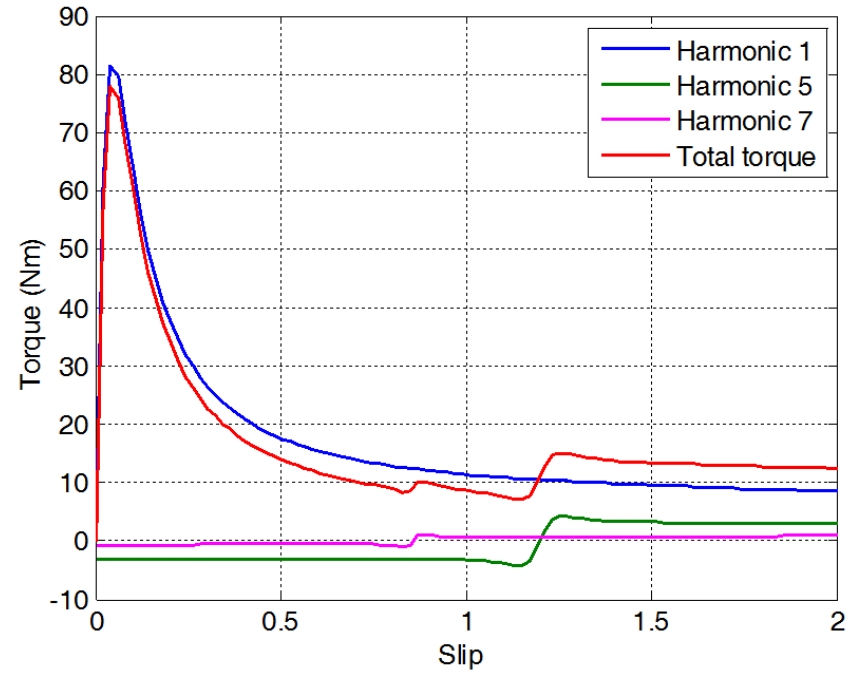

Fig. 16. Torque-slip curve with $5^{\text {th }}$ and $7^{\text {th }}$ harmonic components effects (analytical model).

\section{CONCLUSION}

An accurate analytical model has been developed to compute induced currents within conducting materials placed in the slot of electrical machines. The time-harmonic Helmholtz's equation in polar coordinates has been solved in the slot regions by the technique of separation of variables. The method has been validated by finite element analysis and used for the prediction of the eddy-current induced in the rotor bars of induction motor. In addition, we have shown that the proposed model provides accurate results for the torque-speed curve prediction of induction motor taking into account the space harmonics. 
The analytical model leads to lower computational time than FEM. Therefore, it can be used to investigate rapidly (under linear condition) the influence of the design parameters such as rotor slots dimensions (height and slot opening angle), number of rotor slots, electrical conductivity of the rotor bars and stator winding configuration.

\section{APPENDIX}

For the determination of the integration coefficients, we have to calculate integrals of the form

$$
\begin{aligned}
& f(k, n, i)=\int_{\theta_{i}}^{\theta_{i}+\beta} \cos (n \theta) \cos \left(\frac{k \pi}{\beta}\left(\theta-\theta_{i}\right)\right) d \theta \\
& g(k, n, i)=\int_{\theta_{i}}^{\theta_{i}+\beta} \sin (n \theta) \cos \left(\frac{k \pi}{\beta}\left(\theta-\theta_{i}\right)\right) d \theta \\
& r(n, i)=\int_{\theta_{i}}^{\theta_{i}+\beta} \cos (n \theta) d \theta \\
& s(n, i)=\int_{\theta_{i}}^{\theta_{i}+\beta} \sin (n \theta) d \theta
\end{aligned}
$$

The development of (A.1) and (A.2) gives the following functions that will be used in the expressions of the integration coefficients

- for $k \pi \neq n \beta$

$$
\begin{aligned}
& f(k, n, i)=\frac{-n \beta^{2}\left((-1)^{k} \sin n\left(\beta+\theta_{i}\right)-\sin \left(n \theta_{i}\right)\right)}{k^{2} \pi^{2}-n^{2} \beta^{2}} \\
& g(k, n, i)=\frac{n \beta^{2}\left((-1)^{k} \cos n\left(\beta+\theta_{i}\right)-\cos \left(n \theta_{i}\right)\right)}{k^{2} \pi^{2}-n^{2} \beta^{2}}
\end{aligned}
$$

$$
\begin{aligned}
\text { - for } k \pi & =n \beta \\
f(k, n, i) & =\frac{\beta}{2}\left(\cos \left(n \theta_{i}\right)+\frac{1}{2 k \pi}\left(\sin n\left(\theta_{i}+2 \beta\right)-\sin \left(n \theta_{i}\right)\right)\right) \\
g(k, n, i) & =\frac{\beta}{2}\left(\sin \left(n \theta_{i}\right)-\frac{1}{2 k \pi}\left(\cos n\left(\theta_{i}+2 \beta\right)-\cos \left(n \theta_{i}\right)\right)\right)
\end{aligned}
$$

The development of (A.3) and (A.4) gives the following functions

$$
\begin{gathered}
r(n, i)=\frac{1}{n}\left(\sin \left(n \theta_{i}+n \beta\right)-\sin \left(n \theta_{i}\right)\right) \\
s(n, i)=\frac{1}{n}\left(-\cos \left(n \theta_{i}+n \beta\right)+\cos \left(n \theta_{i}\right)\right)
\end{gathered}
$$

The solution for the seven unknown integration constants can be determined by solving a linear system of equations which can be written in matrix form as

$$
\begin{aligned}
& {[M](X)=(S)} \\
& (X)=\left(\begin{array}{lllllll}
\overline{C_{0}^{i}} & \overline{C_{k}^{i}} & \overline{A_{n}^{I}} & \overline{B_{n}^{I}} & \overline{C_{n}^{I}} & \overline{D_{n}^{I}} & \overline{A_{0}^{I}}
\end{array}\right)^{t}
\end{aligned}
$$

The matrix $[M]$ and the source vector $(S)$ are developed in (A.19) where $N$ and $K$ represent respectively the number of harmonics used in the air-gap and in the rotor bar regions. $Q$ is the number of rotor bars. $I_{Q}$ is the identity matrix of dimension $Q$. A numerical solution of (A.19) can be obtained by using mathematical software (Matlab).

- Expression of the integration constants $\overline{C_{0}^{i}}$ and $\overline{C_{k}^{i}}$ for the $i$-th rotor bar sub-domain

The treatment of (31) yields to the following linear relation

$$
\begin{aligned}
& \overline{C_{0}^{i}}=\overline{A_{0}^{I}} \\
& +\sum_{n=1}^{\infty}\left(\overline{A_{n}^{I}} \frac{R_{2}}{n \beta} \frac{P_{n}\left(R_{2}, R_{3}\right)}{E_{n}\left(R_{2}, R_{3}\right)}+\overline{B_{n}^{I}} \frac{R_{3}}{n \beta} \frac{2}{E_{n}\left(R_{3}, R_{2}\right)}\right) r(n, i) \\
& +\sum_{n=1}^{\infty}\left(\overline{C_{n}^{I}} \frac{R_{2}}{n \beta} \frac{P_{n}\left(R_{2}, R_{3}\right)}{E_{n}\left(R_{2}, R_{3}\right)}+\overline{D_{n}^{I}} \frac{R_{3}}{n \beta} \frac{2}{E_{n}\left(R_{3}, R_{2}\right)}\right) s(n, i)
\end{aligned}
$$

which can be rewritten in vector and matrix form as indicated in the first line of the matrix $[M]$ in (A.19).

The treatment of (32) yields to the following linear relation

$$
\begin{aligned}
& \overline{C_{k}^{i}}=\sum_{n=1}^{\infty}\left(\overline{A_{n}^{I}} \frac{2 R_{2}}{n \beta} \frac{P_{n}\left(R_{2}, R_{3}\right)}{E_{n}\left(R_{2}, R_{3}\right)}+\overline{B_{n}^{I}} \frac{R_{3}}{n \beta} \frac{4}{E_{n}\left(R_{3}, R_{2}\right)}\right) f(k, n, i) \\
& +\sum_{n=1}^{\infty}\left(\overline{C_{n}^{I}} \frac{2 R_{2}}{n \beta} \frac{P_{n}\left(R_{2}, R_{3}\right)}{E_{n}\left(R_{2}, R_{3}\right)}+\overline{D_{n}^{I}} \frac{R_{3}}{n \beta} \frac{4}{E_{n}\left(R_{3}, R_{2}\right)}\right) g(k, n, i)
\end{aligned}
$$

which can be rewritten in vector and matrix form as indicated in the second line of the matrix $[M]$ in (A.19). It is worth noting that the vector $\left(\overline{C_{k}^{i}}\right)_{K Q}$ in (A.19) is of dimension $K \cdot Q$ and takes into account the $Q$ rotor bars.

- Expressions of the integration constants $\overline{A_{n}^{I}}, \overline{B_{n}^{I}}, \overline{C_{n}^{I}}$ and $\overline{D_{n}^{I}}$ for the air-gap sub-domain

The development of (41) and (43) gives

$$
\begin{aligned}
& \overline{B_{n}^{I}}=\left\{\begin{array}{lll}
\mu_{0} J_{m} & \text { for } & n=m p \\
0 & \text { for } & n \neq m p
\end{array}\right. \\
& \overline{D_{n}^{I}}=\left\{\begin{array}{lll}
-j \mu_{0} J_{m} & \text { for } & n=m p \\
0 & \text { for } & n \neq m p
\end{array}\right.
\end{aligned}
$$

where $p$ and $m$ are respectively the number of pole pairs and the $m$-th space harmonics of the current sheet. 
(A.15) and (A.16) can be rewritten in vector and matrix form as indicated in the fourth and the sixth lines of the matrix $[M]$ and vector source $(S)$ in (A.19).

The development of (44) and (45) gives

$$
\begin{aligned}
& \overline{A_{n}^{I}}=\sum_{i=1}^{Q} \overline{C_{0}^{i}} \frac{1}{\pi} \frac{-\alpha J_{1}\left(\alpha R_{2}\right)+\overline{F_{1}} \alpha Y_{1}\left(\alpha R_{2}\right)}{J_{0}\left(\alpha R_{2}\right)-\overline{F_{1}} Y_{0}\left(\alpha R_{2}\right)} r(n, i) \\
& +\sum_{i=1}^{Q} \sum_{k=1}^{\infty} \overline{C_{k}^{i}} \frac{1}{\pi} \frac{J_{k \pi / \beta}^{\prime}\left(\alpha R_{2}\right)-\overline{F_{k}} Y_{k \pi / \beta}^{\prime}\left(\alpha R_{2}\right)}{J_{k \pi / \beta}\left(\alpha R_{2}\right)-\overline{F_{k}} Y_{k \pi / \beta}\left(\alpha R_{2}\right)} f(k, n, i)
\end{aligned}
$$

$$
\begin{aligned}
& \overline{C_{n}^{I}}=\sum_{i=1}^{Q} \overline{C_{0}^{i}} \frac{1}{\pi} \frac{-\alpha J_{1}\left(\alpha R_{2}\right)+\overline{F_{1}} \alpha Y_{1}\left(\alpha R_{2}\right)}{J_{0}\left(\alpha R_{2}\right)-\overline{F_{1}} Y_{0}\left(\alpha R_{2}\right)} s(n, i) \\
& +\sum_{i=1}^{Q} \sum_{k=1}^{\infty} \overline{C_{k}^{i}} \frac{1}{\pi} \frac{J_{k \pi / \beta}^{\prime}\left(\alpha R_{2}\right)-\overline{F_{k}} Y_{k \pi / \beta}^{\prime}\left(\alpha R_{2}\right)}{J_{k \pi / \beta}\left(\alpha R_{2}\right)-\overline{F_{k}} Y_{k \pi / \beta}\left(\alpha R_{2}\right)} g(k, n, i)
\end{aligned}
$$

where $\quad J_{k \pi / \beta}^{\prime}\left(\alpha R_{2}\right)$ means $d J_{k \pi / \beta}(\alpha r) /\left.d r\right|_{R_{2}}$.

(A.17) and (A.18) can be rewritten in vector and matrix form as indicated in the third and the fifth lines of the matrix $[M]$ in (A.19).

The seventh line in (A.19) corresponds to the relation (47).

$\left[\begin{array}{ccccccc}-I_{Q} & 0 & \bar{M}_{Q \times N}^{(13)} & \bar{M}_{Q \times N}^{(14)} & \bar{M}_{Q \times N}^{(15)} & \bar{M}_{Q \times N}^{(16)} & (1)_{Q} \\ 0 & -I_{K Q} & \bar{M}_{K Q \times N}^{(23)} & \bar{M}_{K Q \times N}^{(24)} & \bar{M}_{K Q \times N}^{(25)} & \bar{M}_{K Q \times N}^{(26)} & 0 \\ \bar{M}_{N \times Q}^{(31)} & \bar{M}_{N \times K Q}^{(32)} & -I_{N} & 0 & 0 & 0 & 0 \\ 0 & 0 & 0 & -I_{N} & 0 & 0 & 0 \\ \bar{M}_{N \times Q}^{(51)} & \bar{M}_{N \times K Q}^{(52)} & 0 & 0 & -I_{N} & 0 & 0 \\ 0 & 0 & 0 & 0 & 0 & -I_{N} & 0 \\ (1)^{t} & 0 & 0 & 0 & 0 & 0 & 0\end{array}\right] \times\left[\begin{array}{l}\left(\overline{C_{0}^{i}}\right)_{Q} \\ \left(\overline{C_{k}^{i}}\right)_{K Q} \\ \left(\overline{A_{n}^{I}}\right)_{N} \\ \left(\overline{B_{n}^{I}}\right)_{N} \\ \left(\overline{C_{n}^{I}}\right)_{N} \\ \left(\overline{D_{n}^{I}}\right)_{N} \\ \left(\overline{A_{0}^{I}}\right)_{1}\end{array}\right]=\left[\begin{array}{c}(0)_{Q} \\ (0)_{K Q} \\ (0)_{N} \\ \left(\overline{S_{4}}\right)_{N} \\ (0)_{N} \\ \left(\overline{S_{6}}\right)_{N} \\ (0)_{1}\end{array}\right]$

[11] D. Gerling, and G. Dajaku, "Three-dimensional analytical calculation of induction machines with multilayer rotor structure in cylindrical coordinates," Elec. Eng., vol. 86, no. 4, pp. 199-212, 2004.

[12] M. R. Shah, and S. B. Lee "Rapid analytical optimization of eddycurrent shield thickness for associated loss minimization in electrical machines," IEEE Trans. Ind. Appl., vol. 42, no. 3, pp. 642-649, May/Jun. 2006.

[13] S. A. Swann, and J.W. Salmon, "Effective resistance and reactance of a rectangular conductor placed in a semi-closed slot", proc. IEE, Vol. 110, no. 9, pp. 1656-1662, Sep. 1963.

[14] K. J. Binns, P. J. Lawrenson, and C. W. Trowbridge, The Analytical and numerical solution of electric and magnetic fields, John Wiley \& Sons, pp. 100-102, 1992.

[15] B. Ackermann, and R. Sottek, "Analytical modeling of the cogging torque in permanent magnet motors," Elect. Eng., vol. 78, no. 2, pp. 117-125, Mar. 1994

[16] B. L. J. Gysen, K. J. Meessen, J. J. H. Paulides, and E. A. Lomonova, "General formulation of the electromagnetic field distribution in machines and devices using Fourier analysis," IEEE Trans. Magn., vol. 46, no. 1, pp. 39-52, Jan. 2010.

[17] Z. Q. Zhu, L. J. Wu, and Z.P. Xia, "An accurate subdomain model for magnetic field computation in slotted surface-mounted permanent magnet machines," IEEE Trans. Magn., vol. 46, no. 4, pp. 11001115, Apr. 2010.

[18] T. Lubin, S. Mezani, and A. Rezzoug, "2-D Exact Analytical Model for Surface-Mounted Permanent-Magnet Motors With Semi-Closed Slots," IEEE Trans. Magn., vol. 47, no. 2, pp. 479-492, Feb. 2011.

[19] S. J. Farlow, Partial Differential Equations for Scientists and Engineers. Dover publications, New York, 414 pp, 1993.

[20] D. C. Meeker, "Finite Element Method Magnetics", Version 4.2 (1 April 2009 Build), http://www.femm.info. 